\title{
Re: Robot-assisted laparoscopic radical prostatectomy: assistant's seniority has no influence on perioperative course
}

\author{
Igor Sorokin ${ }^{1} \cdot$ Jessica Nelson ${ }^{1} \cdot$ Noah E. Canvasser ${ }^{2}$
}

Published online: 12 June 2018

๑) Springer-Verlag London Ltd., part of Springer Nature 2018

Keywords Robotic surgery $\cdot$ Bedside assistant $\cdot$ Robot assistant

In the third Journal of Robotic Surgery (JORS) TwitterTM journal club, we discussed Abu-Ghanem and colleague's manuscript on the role of the bedside assistant in robotic surgery [1]. This study specifically looked at the shortterm perioperative outcomes after robotic prostatectomy by an experienced single surgeon. Bedside assistants were separated into either faculty, post-graduate year (PGY) 1-3 residents, and PGY 4-6 residents. Analyzing 106 consecutive cases, the authors found that assistant's seniority had no influence on total operative time, blood loss, complications, or hospital length of stay. An editorial written on this same article by Cadeddu noted that these findings should be reassuring to both the surgeon and the patient [2]. Since the bedside assistant is an integral part of robotic surgery, this topic was further discussed in our journal club [see supplementary table 1].

There was a consensus among journal club participants that bedside assistants do matter in robotic surgery, even in experienced hands. An assistant is not only important for basic tasks like suctioning, irrigating, swapping out instruments, and preventing collisions, they also provide guidance or second opinions if needed (1). A "silent assistant" is knowledgeable about the steps of the operation and can perform tasks without being prompted by the surgeon (2). This may surely offload some of the burden and anxiety of

Electronic supplementary material The online version of this article (https://doi.org/10.1007/s11701-018-0836-z) contains supplementary material, which is available to authorized users.

Igor Sorokin

igsorokin@gmail.com

1 Department of Urology, UT Southwestern Medical Center, 5323 Harry Hines Boulevard, Dallas, TX 75390, USA

2 Department of Urology, UC Davis Medical Center, Sacramento, CA, USA the surgeon. However, as long as a suitable assistant is present, a case should not be cancelled if a trusted assistant is not readily available $(3,4)$. While regular assistants are a nice luxury, making the assistant's job as simple as possible can make the case applicable to any assistant (5).

One interesting aspect of this study was that no advanced practice providers (APPs) were used to assist on the robot. A well-trained APP at the bedside is helpful for trainee guidance and to increase console time $(6,7)$. Of course the tradeoff is decreased trainee bedside experience, which was noted to be a crucial part of learning in laparoscopic surgery as discussed in a previous JORS journal club [3]. In addition, trainees familiar with assisting may have gained important knowledge when it comes to troubleshooting bedside issues (8).

Surgeons may alter their technique based on who is assisting. Surgeons who use a consistent APP may have them perform more complex maneuvers like retracting and placing bulldog clamps that could be challenging for a PGY1 (9). Surgeons may also tailor their approach to clipping, utilizing either an experienced assistant or switch to the robotic clip applier (10). For example, the approach to the neurovascular bundles in a robotic prostatectomy may be affected. Our poll revealed that most participants (67\%) felt they did make adjustments to their technique based on who was assisting (Fig. 1). Nonetheless, the main point of the study as re-iterated by the first author, was that assistants can be safely incorporated into a robotic procedure even when they are less experienced surgeons (PGY1-3) and become "well trained" in robotic surgery regardless of previous practice (11).

This article adds further evidence that residents can be safely utilized for robotic procedures, albeit with an experienced surgeon. A previous study looked at the role of the assistant in robotic partial nephrectomy and found no influence of PGY status on perioperative outcomes [4]. Robotic 
Robotic Surgery

@JRobotSurg

Do you make adjustments to your technique based on who is bed side assisting on the robot? \#robojc

\section{$67 \%$ Yes}

$33 \%$ No

18 votes $\cdot$ Final results

8:51 AM - 2 May 2018

Fig. 1 Twitter poll asking whether surgeons adjust technique based on who is bedside assisting

partial nephrectomy is usually considered more complex for the assistant and likely bears more weight in the debate about the short-term influence of the assistant. On the other hand, robotic prostatectomy involves important long-term quality of life outcomes like continence and potency. While there are certainly many factors at play, it would have been interesting to analyze these outcomes among different assistants. Overall, it is certainly reassuring that immediate outcomes after robotic prostatectomy were not affected by assistant's seniority.

\section{Compliance with ethical standards}

Conflict of interest Igor Sorokin, Jessica Nelson, and Noah Canvasser, declare that they have no conflict of interest.

Ethical approval This article does not contain any studies with human participants or animals performed by any of the authors.

\section{References}

1. Abu-Ghanem Y, Erlich T, Ramon J et al (2017) Robot assisted laparoscopic radical prostatectomy: assistant's seniority has no influence on perioperative course. J Robot Surg 11:305

2. Cadeddu JA (2018) Re: robot assisted laparoscopic radical prostatectomy: assistant's seniority has no influence on perioperative course. J Urol 199:876

3. Canvasser NE, Gahan J, Sorokin I (2018) Re: Time to consider integration of a formal robotic-assisted surgical training program. J Robot Surg 12:199

4. Potretzke AM, Knight BA, Brockman JA et al (2016) The role of the assistant during robot-assisted partial nephrectomy: does experience matter? J Robot Surg 10:129 